\title{
Development, validation, and application of a quantitative volumetric absorptive microsampling-based method in finger prick blood by means of LC-HRMS/MS applicable for adherence monitoring of antipsychotics
}

\author{
Cathy M. Jacobs ${ }^{1} \cdot$ Lea Wagmann ${ }^{1} \cdot$ Markus R. Meyer $^{1}$ \\ Received: 9 September 2020 /Revised: 16 December 2020 / Accepted: 22 December 2020 / Published online: 30 January 2021 \\ (C) The Author(s) 2021
}

\begin{abstract}
Volumetric absorptive microsampling (VAMS), an emerging microsampling technique, is expected to overcome some disadvantages of dried blood spots such as volume inaccuracy and influence of hematocrit (HT). This study aimed to develop and evaluate a VAMS-based strategy for quantification of 13 frequently prescribed antipsychotics in finger prick blood within the scope of adherence monitoring to complement already-established qualitative urine analysis. The final workflow consisted of VAMS tip hydration and subsequent precipitation. Samples were analyzed by using reversed-phase ultra-high-performance liquid chromatography and Orbitrap mass spectrometry operated in parallel reaction monitoring mode. The analytical procedure was successfully validated based on international recommendations at three different HT values $(20 \%, 40 \%, 60 \%)$ for most of the analytes. Selectivity and within/between-run accuracy and precision were in accordance with the recommendations in most cases. Internal standard-normalized matrix factor met recommended criteria for all analytes at HT 40\%. For the HT values of $20 \%$ and $60 \%$, only four substances did not meet the criteria. Dilution integrity was given for all substances, except for olanzapine, allowing a quantification over the whole therapeutic range of selected antipsychotics. Long-term stability in VAMS tips was tested and revealed degradation of five antipsychotic drugs after 1 week of storage at $24{ }^{\circ} \mathrm{C}$. A proof of concept of the applicability of the method was obtained by quantification of a selection of the 13 antipsychotic drugs in VAMS tips and matched plasma samples. Results were coherent between matrices. Thus, VAMS was shown to be a promising alternative for adherence monitoring of at least the investigated antipsychotics.
\end{abstract}

Keywords Adherence monitoring $\cdot$ Microsampling $\cdot$ VAMS $\cdot$ Antipsychotics $\cdot$ LC-HRMS

\section{Introduction}

A combination of antipsychotics and psychotherapy is often used to treat psychotic symptoms caused for example by schizophrenia or bipolar disorders [1,2], but there is a wide interpatient variability concerning the response to the therapy. It is also well known that patients suffering from psychotic symptoms often show low adherence to drug therapy [3-6].

Markus R. Meyer

markus.meyer@uks.eu

1 Department of Experimental and Clinical Toxicology, Institute of Experimental and Clinical Pharmacology and Toxicology, Center for Molecular Signaling (PZMS), Saarland University,

Homburg, Germany
Bohlken et al. came to the conclusion after reviewing a database that only about $60 \%$ of schizophrenia and bipolar disorder patients are expected to be adherent [7]. Consequences of non-adherence are poor or nonresponse to therapy and may then result in an increase of disease burden, inpatient admission rate, suicide rate, and treatment costs $[4,8]$. Currently, analytical adherence monitoring is usually performed using traditional matrices like plasma or serum obtained from venous whole blood [9-11]. However, venous whole blood sampling procedure can cause discomfort and anxiety, especially amongst the psychiatric population and can only be performed by trained personnel. Alternative sampling methods such as microsampling may overcome these disadvantages. One of the most popular methods, first described in 1963, is sampling of dried blood spots (DBS) [12]. DBS consist of small drops of capillary blood usually with unknown volume soaked on 
filter paper [12]. The use of DBS is promoted as easy, costeffective, minimally invasive, and as a suitable home sampling technique. DBS were used for therapeutic drug monitoring (TDM) of antipsychotics amongst others [13, 14]. However, DBS have also some drawbacks like hematocrit (HT) effects and volume inaccuracy [15]. Therefore, further alternatives like volumetric absorptive microsampling (VAMS) have been developed. VAMS devices consist of porous hydrophilic tips attached to a plastic sample handler, which can absorb different defined volumes of finger prick blood (FPB) by wicking (see Supplementary Information (ESM) Fig. S1) [16, 17]. VAMS are expected to maintain the advantages of DBS and to overcome their limitations. VAMS has already been used for monitoring of $\mathrm{HbA1C}$ [18], in metabolomic studies [19], and for screening of plasma, urine, and oral fluid for drugs of abuse [20, 21]. Furthermore, VAMS was used for TDM and determination of pharmacokinetic parameters [22]. Recently, Stern et al. published a method using VAMS for the quantification of psychiatric drugs including some antipsychotics [23]. So far and to the best of our knowledge, no research article demonstrating the feasibility of VAMS for adherence monitoring was published. Therefore, the aim of this study was to develop and evaluate a VAMS-based strategy for adherence monitoring of antipsychotics in FPB by using drug concentrations to complement qualitative urine analysis [24]. Analysis should be done by means of LC-HRMS/MS and the whole quantitative workflow should be validated in accordance with international guidelines [25, 26]. Validation included the evaluation of an HT range from 20 to $60 \%$ and a quantitative comparison of VAMS to plasma samples gained from matched whole blood. The 13 most frequently prescribed antipsychoticsamisulpride, aripiprazole, cyamemazine, clozapine, haloperidol, melperone, olanzapine, paliperidone, pipamperone, promethazine, prothipendyl, quetiapine, and risperidoneshould be included making the method suitable for adherence monitoring as complement to urine analysis and demonstrating its potential for TDM.

\section{Experimental}

\section{Chemicals and other materials}

Quetiapine was purchased from Astra Zeneca, Macclesfield (UK); promethazine from Bayer, Leverkusen (Germany); paliperidone and risperidone from Janssen, Beerse (Belgium); amisulpride, melperone, and prothipendyl from LGC, Luckenwalde (Germany); clozapine from Novartis Pharma, Wehr (Germany); haloperidol from Siegfried, Säckingen (Germany); and aripiprazole, cyamemazine, and olanzapine from Sigma-Aldrich, St. Louis (USA). Trimipramine- $d_{3}$ was purchased from LGC, Wesel
(Germany). All other chemicals (LC-MS grade or analytical grade) were from VWR, Darmstadt (Germany). Mitra VAMS with a $10-\mu \mathrm{L}$ absorbing tip were purchased from Neoteryx, Torrance (USA). Blank EDTA blood used for development and validation of the procedure was collected from drug-free healthy volunteers after obtaining written informed consent. Blood samples for applicability studies were submitted to the authors' laboratory for regular toxicological analysis and handled according to the institutional protocol and regulations concerning data privacy and sample handling.

\section{Calibrators, quality controls, internal standards, and preparation of VAMS}

Stock solutions of each compound were prepared at a concentration of $1 \mathrm{mg} / \mathrm{mL}$ in methanol, except for aripiprazole, olanzapine, and prothipendyl, which were prepared in DMSO. The internal standard (IS) solution contained $0.005 \mathrm{mg} / \mathrm{mL}$ trimipramine- $\mathrm{d}_{3}$ in methanol. Calibrator and quality controle (QC) working solutions were prepared in methanol (stock solution A) or DMSO (stock solution B). The final concentration in whole blood used to load VAMS tips is shown in Table 1. Calibrator and QC samples were obtained by adding $10 \mu \mathrm{L}$ of each working solution to $380 \mu \mathrm{L}$ blank human whole EDTA blood. For the preparation of the different concentrations, the spiked solution did not exceed $5 \%$ of the total matrix volume in order not to influence the composition of the matrix [26]. The preparation of different target HT values was achieved by removing or adding plasma after centrifugation for $11 \mathrm{~min}$ at $9660 \times \mathrm{g}[27,28]$.

Whole blood was incubated for $30 \mathrm{~min}$ at $37{ }^{\circ} \mathrm{C}$ and $1500 \mathrm{rpm}$ to allow plasma-protein binding and diffusion into red blood cells. Afterwards, VAMS tips were held onto the surface of whole blood until they turned completely red with an additional waiting time of $2 \mathrm{~s}$ [16]. VAMS tips were dried for $3 \mathrm{~h}$ at room temperature $\left(24^{\circ} \mathrm{C}\right)$ before sample preparation [29]. Concentrations of standard solutions used for standard addition procedure in plasma are shown in the ESM in Table S1. All solutions were stored at $-20{ }^{\circ} \mathrm{C}$ in amber glass vials.

\section{VAMS sample preparation}

The sample preparation was based on a method published by D'Urso et al. with some adjustments [30]. Dried VAMS tips were stripped into $2-\mathrm{mL}$ reaction tubes. Volumes of $90 \mu \mathrm{L}$ purified water and $10 \mu \mathrm{L}$ IS solution were added. Samples were shaken for $15 \mathrm{~min}$ at $1500 \mathrm{rpm}$ and $37^{\circ} \mathrm{C}$. Afterwards, $200 \mu \mathrm{L}$ of acetonitrile $(\mathrm{ACN})$ was added and samples were shaken for $30 \mathrm{~min}$ at $1500 \mathrm{rpm}$ and room temperature $\left(24{ }^{\circ} \mathrm{C}\right)$ followed by $10 \mathrm{~min}$ of centrifugation at $15,000 \times \mathrm{g}$ and at $-10^{\circ} \mathrm{C}$. Finally, the supernatant was transferred into LC vials and a volume of $20 \mu \mathrm{L}$ was injected onto the LC-HRMS/MS system, and analyzed as described below. 
Table 1 Final concentrations ( $\mathrm{ng} / \mathrm{mL}$ ) of analytes in whole blood used to load calibrator (Cal) VAMS-tips and quality control (QC) VAMS-tips as well as weighting factors used for linear regression. $L L O Q$ lower limit of quantification, $U L O Q$ upper limit of quantification

\begin{tabular}{lllllllllllll}
\hline Analyte & Cal 1 LLOQ & Cal 2 & Cal 3 & Cal 4 & Cal 5 & Cal 6 & Cal 7 & Cal 8 ULOQ & QC low & QC medium & QC high & Weighting \\
\hline Amisulpride & 50 & 85 & 100 & 200 & 400 & 600 & 800 & 1000 & 150 & 500 & 850 & $1 / x^{2}$ \\
Aripiprazole & 50 & 85 & 100 & 200 & 400 & 600 & 800 & 1000 & 150 & 500 & 850 & $1 / x^{2}$ \\
Clozapine & 50 & 85 & 100 & 200 & 400 & 600 & 800 & 1000 & 150 & 500 & 850 & $1 / x^{2}$ \\
Cyamemazine & 0.5 & 0.85 & 1 & 2 & 4 & 6 & 8 & 10 & 1.5 & 5 & 8.5 & $1 / x$ \\
Haloperidol & 0.5 & 0.85 & 1 & 2 & 4 & 6 & 8 & 10 & 1.5 & 5 & 8.5 & Equal \\
Melperone & 5 & 8.5 & 10 & 20 & 40 & 60 & 80 & 100 & 15 & 50 & 85 & $1 / x^{2}$ \\
Olanzapine & 5 & 8.5 & 10 & 20 & 40 & 60 & 80 & 100 & 15 & 50 & 85 \\
Paliperidone & 5 & 8.5 & 10 & 20 & 40 & 60 & 80 & 100 & 15 & 50 & 85 \\
Pipamperone & 50 & 85 & 100 & 200 & 400 & 600 & 800 & 1000 & 150 & 500 & 850 & $1 / x^{2}$ \\
Promethazine & 5 & 8.5 & 10 & 20 & 40 & 60 & 80 & 100 & 15 & 50 & 85 & $1 / x^{2}$ \\
Prothipendyl & 0.5 & 0.85 & 1 & 2 & 4 & 6 & 8 & 10 & 1.5 & 5 & 8.5 & $1 / x$ \\
Quetiapine & 50 & 85 & 100 & 200 & 400 & 600 & 800 & 1000 & 150 & 500 & 850 & $1 / x^{2}$ \\
Risperidone & 0.5 & 0.85 & 1 & 2 & 4 & 6 & 8 & 10 & 1.5 & 5 & 8.5 & $1 / x^{2}$ \\
\hline
\end{tabular}

\section{Plasma sample preparation}

Concentrations as well as addition steps used for standard addition procedure are shown in the ESM Tables S1 and S2 and extraction was based on a slightly modified procedure published by Montenarh et al. [31]. Briefly, $300 \mu \mathrm{L}$ plasma was mixed with $300 \mu \mathrm{L}$ of Soerensen's buffer (pH 7.4) and $20 \mu \mathrm{L}$ IS solution and then spiked with addition solutions. For liquid-liquid extraction, $700 \mu \mathrm{L}$ ethylacetate:diethylether $(50: 50, \mathrm{v} / \mathrm{v})$ was added and samples were shaken for $5 \mathrm{~min}$, $1500 \mathrm{rpm}$ at room temperature $\left(24^{\circ} \mathrm{C}\right)$ before centrifugation $(5 \mathrm{~min}, 18,407 \times \mathrm{g}$ ). The supernatant was transferred to a vial and evaporated under a gentle flow of nitrogen at $40{ }^{\circ} \mathrm{C}$. The remaining aqueous layer was mixed with $150 \mu \mathrm{L} \mathrm{NaOH}(1 \mathrm{M})$ and $700 \mu \mathrm{L}$ ethylacetate:diethylether $(50: 50, \mathrm{v} / \mathrm{v})$ and shaken for $5 \mathrm{~min}$ at $1500 \mathrm{rpm}$ at room temperature $\left(24{ }^{\circ} \mathrm{C}\right)$ before centrifugation $(5 \mathrm{~min}, 18,407 \times \mathrm{g}$ ). The supernatant was transferred to the previously used vial and also evaporated under a gentle flow of nitrogen at $40{ }^{\circ} \mathrm{C}$. The extracts were finally reconstituted in $100 \mu \mathrm{L}$ of methanol and $20 \mu \mathrm{L}$ was injected onto the LC-HRMS/MS system as described below.

\section{Instrumental settings}

All samples were analyzed using a Thermo Fisher Scientific (TF, Dreieich, Germany) Dionex UltiMate 3000 consisting of a degasser, a quaternary pump, a DL W2 wash system, and an HTC PAL autosampler (CTC Analytics AG, Zwinger, Switzerland), coupled to a TF Q-Exactive system equipped with a heated electrospray ionization (HESI)-II source. Mass calibration was done prior to analysis according to the manufacturer's recommendations using external mass calibration. Gradient elution was performed on a TF Accucore phenyl- hexyl column $(100 \mathrm{~mm} \times 2.1 \mathrm{~mm}, 2.6-\mu \mathrm{m}$ particle size $)$. The mobile phase A consisted of $2 \mathrm{mM}$ aqueous ammonium formate containing formic acid $(0.1 \%, \mathrm{v} / \mathrm{v}, \mathrm{pH} 3)$, and mobile phase $\mathrm{B}$ consisted of $2 \mathrm{mM}$ aqueous ammonium formate with acetonitrile:methanol $(50: 50, \mathrm{v} / \mathrm{v})$ plus formic acid $(0.1 \%$, $\mathrm{v} / \mathrm{v})$, and water $(1 \%, \mathrm{v} / \mathrm{v})$. The flow rate was set at $750 \mu \mathrm{L} /$ $\mathrm{min}$. The gradient was programmed as follows: $0-1 \mathrm{~min}$ from $100 \%$ A to $95 \%$ A, $1-4.2$ min to $90 \%$ A, $4.2-13.5 \mathrm{~min}$ to $85 \%$ A, 13.5 to $18 \mathrm{~min}$ to $65 \% \mathrm{~A}, 18-19 \mathrm{~min}$ to $1 \% \mathrm{~A}, 19-21.8 \mathrm{~min}$ hold $1 \% \mathrm{~A}, 21.8 \mathrm{~min}$ to $25 \mathrm{~min}$ hold $100 \% \mathrm{~A}$. Chromatography was performed at $70{ }^{\circ} \mathrm{C}$. The HESI-II source conditions were as follows: ionization mode, positive; sheath gas flow rate, 60 arbitrary units (AU); auxiliary gas flow rate, $10 \mathrm{AU}$; spray voltage, $4.00 \mathrm{kV}$; auxiliary gas heater temperature, $360{ }^{\circ} \mathrm{C}$; ion transfer capillary temperature, $320^{\circ} \mathrm{C}$; and Slens RF level, 60.0.

Mass spectrometry was performed using parallel reaction monitoring (PRM) with an inclusion list containing masses of interest in defined time windows. The settings for PRM data acquisition were as follows: resolution, 17,500; microscans, 1 ; automatic gain control (AGC) target, 2e5; maximum injection time (IT), $250 \mathrm{~ms}$; scan range, $\mathrm{m} / \mathrm{z}, 170$ to 930 ; isolation window, $2.0 \mathrm{~m} / \mathrm{z}$; HCD with stepped normalized collision energy (NCE), 17.5, 35, and 52.5\%; spectrum data type, profile. TF Xcalibur Qual Browser software version 2.2 was used for data handling. Precursor ion masses $(\mathrm{m} / \mathrm{z})$ used for the inclusion list are represented in the ESM Table S3.

\section{Method validation}

The validation was performed according to published recommendations including the European Medicines Agency (EMA) Guideline on Bioanalytical Method Validation and 
the International Association of Therapeutic Drug Monitoring and Clinical Toxicology (IATDMCT) guideline for development and validation of dried blood spot-based methods for therapeutic drug monitoring $[25,26]$. The statistical evaluation of the peak area ratios of analytes versus IS was performed using Microsoft Excel version 16 (Microsoft, Redmond, USA) and TF Xcalibur Quan Browser version 2.2.

For selectivity testing, 13 blank matrices from individual donors were analyzed in PRM mode for possible peak interferences of analytes or IS. These were divided in 10 drug-free FPB donors and three samples were loaded with EDTA blood-containing drugs but no antipsychotics. For carryover testing, a blank matrix sample was injected after analysis of the highest calibrator $(n=3)$. Interfering signals of analytes in blank matrix should be less than $20 \%$ of the lower limit of quantification (LLOQ) and less than 5\% of the IS for selectivity and carry-over testing.

Three calibration ranges were defined consisting of eight calibration points, each. Calibrators were prepared by spiking blank matrix with different calibrator solutions (final matrix concentrations; see Table 1). Extraction was performed as described before. All LLOQ and upper limits of quantification (ULOQ) are shown in Table 1. Different weighting factors (equal, $1 / x$, or $1 / x^{2}$ ) for linear regression were tested by fitting three curves of each analyte. The suitability of the models was evaluated using the square sum of residuals (SSR) by choosing the model with the lowest SSR. All calculations were performed using KNIME (www.knime.org) and R snippets (www.r-projects.org). Back-calculated concentrations of calibrators should be within $\pm 15 \%$ of the nominal value $(\mathrm{LLOQ} \pm 20 \%$ ). If criteria are not met, the calibration standard should be rejected for regression analysis. At least $75 \%$ of the calibration standards must fulfill criteria.

Accuracy and precision of the quantification results were determined for the LLOQ, QC low, QC medium, and QC high levels listed in Table 2. Therefore, QC samples were spiked with separately prepared stock solutions from calibration standards. QC sample concentrations were back-calculated via the calibration curves and compared with their nominal values. The evaluation was performed for values of five sample replicates obtained within a single run (within-run accuracy and precision) and in three different runs (between-run accuracy and precision). For positive assessment of accuracy, mean concentrations should be within $\pm 15 \%$ of the nominal values $( \pm 20 \%$ for LLOQ). For a positive assessment of precision, the coefficient of variation (CV) should be within 15\% (20\% for LLOQ).

Dilution integrity of the final extract was determined by spiking blank samples $(n=5)$ at 2.5 times higher than the highest calibrator. Extracts were diluted 1:5 with IS-spiked ACN or processed blank matrix containing IS [26]. Since prothipendyl shows a broad therapeutic range (ESM Table S4), two additional dilution factors were tested for this analyte. An extracted sample stemming from a blood concentration of $100 \mathrm{ng} / \mathrm{mL}$ prothipendyl was diluted 1:10 and 1:20 with either IS-spiked ACN or processed blank matrix containing IS. All samples were analyzed, and accuracy and precision were determined.
Table 2 Within- and between-day accuracy (A) and precision (P) of the LLOQ, QC low, QC medium, and QC high. Dilution integrity with acetonitrile containing IS (ACN) or with processed blank matrix containing
IS (matrix). 1:5 dilution of a 2.5 higher concentration than the highest calibrator. $L L O Q$ lower limit of quantification, $Q C$ quality control, $I S$ internal standard; mid medium

\begin{tabular}{|c|c|c|c|c|c|c|c|c|c|c|}
\hline \multirow[t]{3}{*}{ Analyte } & \multicolumn{10}{|c|}{ Relative mean concentration (A), \% (CV (P), \%) } \\
\hline & \multicolumn{4}{|c|}{ Within-day } & \multicolumn{4}{|c|}{ Between-day } & \multicolumn{2}{|l|}{ Dil 1:5 } \\
\hline & LLOQ & QC low & QC mid & QC high & LLOQ & QC low & QC mid & QC high & $\mathrm{ACN}$ & Matrix \\
\hline Amisulpride & $106(8)$ & $102(8)$ & $93(6)$ & $107(7)$ & $105(8)$ & $102(11)$ & $95(6)$ & $104(11)$ & $69(9)$ & $99(5)$ \\
\hline Aripiprazole & $100(2)$ & $100(9)$ & $109(5)$ & $97(4)$ & $99(10)$ & $106(8)$ & $105(5)$ & $100(8)$ & $67(7)$ & $105(7)$ \\
\hline Clozapine & $97(8)$ & $107(8)$ & $102(6)$ & $115(6)$ & $100(6)$ & $106(7)$ & $103(5)$ & $111(8)$ & 77 (7) & $115(4)$ \\
\hline Cyamemazine & $90(13)$ & $95(9)$ & $88(7)$ & $90(7)$ & 115 (27) & $87(17)$ & $84(7)$ & $90(9)$ & $56(12)$ & $90(7)$ \\
\hline Haloperidol & $105(15)$ & $95(9)$ & $100(5)$ & $103(5)$ & $106(17)$ & $101(12)$ & $98(7)$ & $104(11)$ & $80(12)$ & $106(9)$ \\
\hline Melperone & $107(4)$ & $101(8)$ & $96(7)$ & $98(7)$ & $97(10)$ & $96(9)$ & $95(6)$ & $101(10)$ & $61(9)$ & $89(7)$ \\
\hline Olanzapine & $89(14)$ & $101(11)$ & $98(6)$ & $100(2)$ & $92(11)$ & $98(11)$ & $99(6)$ & $101(10)$ & $38(13)$ & $50(12)$ \\
\hline Paliperidone & $96(8)$ & $110(13)$ & $102(8)$ & $109(5)$ & $102(9)$ & 109 (11) & $104(6)$ & 109 (10) & $71(8)$ & $107(4)$ \\
\hline Pipamperone & $96(7)$ & $113(11)$ & $100(6)$ & $111(7)$ & 99 (7) & $111(9)$ & $106(7)$ & $110(9)$ & $65(8)$ & $110(3)$ \\
\hline Promethazine & $97(8)$ & $104(4)$ & $97(6)$ & $104(9)$ & $101(11)$ & $102(7)$ & $96(6)$ & 105 (10) & $65(11)$ & $97(8)$ \\
\hline Prothipendyl & $120(10)$ & $97(12)$ & $102(9)$ & $100(9)$ & $111(38)$ & $98(12)$ & $106(9)$ & $103(10)$ & $70(13)$ & $104(8)$ \\
\hline Quetiapine & $101(3)$ & $105(10)$ & $96(4)$ & $105(3)$ & $108(14)$ & $106(10)$ & $100(6)$ & $106(12)$ & $72(6)$ & $110(4)$ \\
\hline Risperidone & $98(15)$ & $95(8)$ & $86(2)$ & $91(4)$ & 103 (12) & $97(11)$ & $89(5)$ & $96(11)$ & $64(7)$ & 94 (4) \\
\hline
\end{tabular}


The matrix factor (MF) and the recovery (RE) were investigated at low and high QC levels for six blank matrix samples of individual donors. VAMS tips were prepared with whole blood (EDTA) of HT 40\%. Additionally, three blank matrix samples of individual donors were prepared at HT $20 \%$ and HT $60 \%$ each. The MF was calculated by the ratio of the peak area in the presence of matrix (blank matrix spiked after extraction) to the peak area in the absence of matrix (pure analyte solution). The IS-normalized MF was calculated by dividing the MF of the analytes by the MF of the IS. The RE was calculated by the ratio of the peak area extracted with the matrix (blank matrix spiked before extraction) to the peak area in the presence of matrix (blank matrix spiked after extraction). The CV of the IS-normalized MF and the RE should be within $15 \%$.

The stability of the stock solutions A and B was tested over a time of 10 weeks $(n=3)$. Furthermore, autosampler stability $\left(24 \mathrm{~h}, 10^{\circ} \mathrm{C}\right)$ of processed samples and long-term stability $(1$ and 2 weeks, $24^{\circ} \mathrm{C}$ ) in the test device were investigated $(n=4)$ using the low and high QC levels. Deviations between obtained and nominal concentrations of QCs should be within \pm $15 \%$ when analyzed immediately after preparation and after evaluated storage conditions using a freshly prepared calibration curve.

\section{Proof of concept}

Matched human plasma samples and VAMS tips soaked with EDTA blood of 10 individuals were analyzed. All samples were submitted to the authors' laboratory for regular toxicological analysis.

\section{Results and discussion}

Assessing adherence based on serum drug concentrations to complement urine analysis is expected to be more accurate than urine analysis alone, particularly when using the socalled dose-related concentration approach as proposed, e.g., by Ritscher et al. [24]. In general, drugs with low bioavailability, low renal excretion, or underlying extensive metabolism may be underestimated concerning their adherence when using qualitative urine analysis alone. Therefore, we aimed to develop a strategy for the simultaneous quantification of the 13 most frequently prescribed antipsychotics in FPB using VAMS with a particular focus on adherence monitoring. Paliperidone (9-hydroxyrisperidone) can be prescribed as a drug but is also the active metabolite of risperidone. Aripiprazole, clozapine, and quetiapine also have active metabolites; however, none of them can be prescribed as a drug itself. For a correct assessment of adherence, the quantification of active metabolites is not necessary. However, this might be a limitation point if the method is used for TDM.
Figures S2 and S3 (ESM) show the chromatographic separation of the antipsychotics at the LLOQ level and in a patient sample within $20 \mathrm{~min}$, respectively. The long run time of 20 min (including the equilibration step of then in total $25 \mathrm{~min}$ ) was acceptable as the method could be integrated into our laboratory routine. The aim was to achieve sufficient separation without changing the column type or composition of mobile phases and to retain the standard setup. Furthermore, adherence monitoring is normally no urgent analysis, and therefore a total runtime of $25 \mathrm{~min}$ was acceptable. An injection volume of $20 \mu \mathrm{L}$ was chosen since it provided better sensitivity compared to a volume of $10 \mu \mathrm{L}$. This in turn led to peak-decompression, which was tolerated and had no negative impact on analytical results. For identification, full $\mathrm{MS}^{2}$ spectra were compared to a database [32] and the peak area ratios of the analyte-specific fragment ion in $\mathrm{MS}^{2}$ (ESM Table S3) and those of the IS were used for quantification. For analysis of human samples, each analytical batch consisted of a zero-sample containing IS but no antipsychotics, eight calibration standards, three QC levels, and the patient samples.

The IS trimipramine- $\mathrm{d}_{3}$ did not coelute with the analytes. No interfering signals from endogenous compounds or other drugs (listed in ESM Table S5) as well as no IS carry-over could be observed. However, carry-over was occasionally observed for cyamemazine, haloperidol, and olanzapine especially after injection of concentrations above the therapeutic range. Hence, samples following a high concentration of those drugs should be reanalyzed after a washing run with extracted blank matrix.

A linear calibration model could be used for all analytes after testing different weighting factors (equal, $1 / x, 1 / x^{2}$; see Table 1). Results for the within- and between-day accuracy and precision are shown in Table 2. For the within-day accuracy, all analytes had a mean concentration within $\pm 15 \%$ (LLOQ $\pm 20 \%$ ) of the nominal concentration and the withinday precision did not exceed a CV of $15 \%$. However, for between-day accuracy and precision, cyamemazine only met required validation parameters at the high $\mathrm{QC}$ level and prothipendyl exceeded precision at the LLOQ. Anyhow, the lowest therapeutic concentration of prothipendyl is already covered by the QC low level. Consequently, the QC low level should be used as cutoff concentration for the quantification of prothipendyl. Cyamemazine failed the validation in this assay.

Dilution integrity was tested by dilution (1:5) of a processed sample, having a concentration 2.5 times higher than the highest calibrator, either with IS-spiked ACN (trimipramine- $\mathrm{d}_{3}, 0.166 \mathrm{mg} / \mathrm{L}$ ) or with processed blank matrix containing IS. Using IS-spiked ACN for dilution, none of the analytes passed validation. However, if the final extract was diluted with processed blank matrix containing IS, all analytes passed validation except olanzapine. Anyway, the therapeutic range of olanzapine is completely covered by the calibration 
range. For prothipendyl, two additional dilution factors (1:10 and 1:20) were tested. Again, validation parameters were only fulfilled when processed blank matrix containing IS was used (see ESM Table S6).

Determined IS-normalized MFs and CVs are given in Table 3. At different HT values, MFs varied between 0.77 and 1.28. However, these effects were found to be reproducible with CVs within 15\% except for haloperidol (QC low HT $20 \%$ and HT 60\%), olanzapine (QC low and high HT 60\%), and prothipendyl (QC low HT 20\% and HT 60\%). Thus, the MF was reproducible for all analytes at HT $40 \%$. Higher CVs at HT $20 \%$ and HT $60 \%$ for haloperidol, olanzapine, and prothipendyl should not play a role for normal HT fluctuation of healthy patients.

$\mathrm{RE}$ values of the analytes of low and high QC in VAMS tips at different HT values are summarized in Table 3. The RE was found to be reproducible, even at low HT values, with CVs within 15\% except for cyamemazine (QC low HT 60\%), olanzapine (QC low HT 40\% and HT 60\%; QC high HT $40 \%$ ), and prothipendyl (QC low HT 20\%). However, CVs never exceeded $21 \%$. Only for a few antipsychotics, the nominal RE value at HT $20 \%$ and $60 \%$ differed from the HT $40 \%$ value by more than $15 \%$. Greater nominal RE deviations at the low HT values were observed for amisulpride (QC high HT 20\%), haloperidol (QC low HT 20\% and QC high HT 60\%), paliperidone (QC low HT 20\%), prothipendyl (QC low HT $20 \%$ ), and risperidone (QC low HT 60\%). Only in the case of haloperidol (21\%) and prothipendyl (30\%), nominal RE deviation exceeded $20 \%$. In summary, the present method allowed the quantification of amisulpride, aripiprazole, clozapine, haloperidol, melperone, olanzapine, paliperidone, pipamperone, promethazine, prothipendyl, quetiapine, and risperidone over their complete therapeutic range (ESM Table S4) in whole blood (HT 40\%) using VAMS devices. For prothipendyl, concentration exceeding the QC high can be quantified since dilution integrity was given for three dilution factors. Only cyamemazine failed validation in between-day accuracy and precision.

Stock solutions were stable over 10 weeks at $-20{ }^{\circ} \mathrm{C}$ in amber glass vials. Results of short- and long-term stability are given in ESM Table S7. After storage of processed samples in the autosampler for $24 \mathrm{~h}$ at $10^{\circ} \mathrm{C}$, no analyte showed a degradation over $15 \%$ of the nominal concentration for the low and high QC levels. This is of advantage if long-lasting analytical series are planned. However, autosampler stability can only be guaranteed for $24 \mathrm{~h}$, based on these experiments. Hence, the duration of a batch should not exceed this period of time. However, not all analytes are long-term stable in the device. After 1 -week storage at $24{ }^{\circ} \mathrm{C}$, cyamemazine, melperone, olanzapine, promethazine, and prothipendyl showed degradation over $15 \%$ of nominal concentration. Haloperidol was stable for 1 week and amisulpride, aripiprazole, clozapine, paliperidone, pipamperone, quetiapine, and risperidone were stable for 2 weeks in the sample device at $24{ }^{\circ} \mathrm{C}$. The instability of olanzapine and promethazine in whole blood at $20^{\circ} \mathrm{C}$ was already described but melperone was stable over 10 weeks at $20^{\circ} \mathrm{C}$ in whole blood [33]. Good stability of amisulpride, aripiprazole, clozapine, haloperidol, paliperidone, pipamperone, quetiapine, and risperidone was already described for DBS [14, 34, 35]. Proof

Table 3 Internal standard (IS)-normalized matrix factors (MF), recovery (RE), and coefficients of variation (CVs) of the analytes of low- and highquality controls (QC) for VAMS at different hematocrit (HT) values ( $n=6$ at HT 40\%; $\mathrm{n}=3$ at HT $20 \%$ and HT $60 \%)$

\begin{tabular}{|c|c|c|c|c|c|c|c|c|c|c|c|c|}
\hline \multirow[t]{3}{*}{ Analyte } & \multicolumn{6}{|c|}{ IS-normalized MF (CV, \%) } & \multicolumn{6}{|c|}{$\mathrm{RE}, \%(\mathrm{CV}, \%)$} \\
\hline & \multicolumn{3}{|l|}{ QC low } & \multicolumn{3}{|l|}{ QC high } & \multicolumn{3}{|l|}{ QC low } & \multicolumn{3}{|l|}{ QC high } \\
\hline & HT $20 \%$ & HТ $40 \%$ & HT $60 \%$ & HT $20 \%$ & HT $40 \%$ & HT $60 \%$ & HT $20 \%$ & HT $40 \%$ & HТ $60 \%$ & HT $20 \%$ & HT $40 \%$ & HT $60 \%$ \\
\hline Amisulpride & $0.85(7)$ & $0.84(6)$ & $0.98(7)$ & $0.83(7)$ & $0.86(7)$ & $0.87(10)$ & $138(8)$ & $123(6)$ & $132(13)$ & $146(7)$ & $130(4)$ & $132(3)$ \\
\hline Aripiprazole & $0.97(5)$ & $1.02(8)$ & $1.15(2)$ & $1.05(4)$ & $1.05(8)$ & $1.08(10)$ & $131(8)$ & $123(8)$ & $117(4)$ & $128(4)$ & $118(5)$ & $110(6)$ \\
\hline Clozapine & $0.95(6)$ & $0.92(6)$ & $1.06(4)$ & $0.91(5)$ & $0.91(6)$ & $0.95(9)$ & $111(5)$ & $114(9)$ & $109(3)$ & $117(4)$ & $105(7)$ & $102(6)$ \\
\hline Cyamemazine & $1.24(9)$ & $1.28(12)$ & $1.26(1)$ & $1.14(8)$ & $1.09(8)$ & $1.10(6)$ & $49(13)$ & $52(10)$ & $63(18)$ & $68(9)$ & $68(8)$ & $58(4)$ \\
\hline Haloperidol & $0.94(20)$ & $0.99(14)$ & $1.08(33)$ & $0.93(8)$ & $0.95(3)$ & $0.92(5)$ & $71(2)$ & $93(12)$ & $103(14)$ & $80(12)$ & $75(13)$ & $91(7)$ \\
\hline Melperone & $0.85(4)$ & $0.85(5)$ & $0.95(6)$ & $0.89(7)$ & $0.89(5)$ & $0.94(8)$ & $63(15)$ & $67(11)$ & $65(15)$ & $54(10)$ & $60(11)$ & $61(6)$ \\
\hline Olanzapine & $0.77(10)$ & $0.81(10)$ & $0.93(20)$ & $0.86(7)$ & $0.85(7)$ & $0.94(17)$ & $26(5)$ & $13(18)$ & $16(21)$ & $34(15)$ & $26(18)$ & $20(14)$ \\
\hline Paliperidone & $0.88(6)$ & $0.87(7)$ & $1.04(12)$ & $0.90(4)$ & $0.91(5)$ & $0.95(11)$ & $112(3)$ & $132(9)$ & $138(12)$ & $136(3)$ & $126(8)$ & $123(8)$ \\
\hline Pipamperone & $0.99(4)$ & $1.00(5)$ & $1.12(4)$ & $0.99(7)$ & $0.98(6)$ & $1.01(9)$ & $114(8)$ & $114(7)$ & $123(8)$ & $115(3)$ & $115(4)$ & $112(5)$ \\
\hline Promethazine & $0.92(5)$ & $0.92(6)$ & $1.01(8)$ & $0.97(4)$ & $0.96(5)$ & $1.02(9)$ & $60(12)$ & $57(14)$ & $56(11)$ & $59(6)$ & $55(7)$ & $52(7)$ \\
\hline Prothipendyl & $0.96(18)$ & $1.10(14)$ & $1.06(22)$ & $0.96(4)$ & $0.91(11)$ & $0.99(6)$ & $32(20)$ & $62(16)$ & $63(4)$ & $45(9)$ & $46(15)$ & $39(6)$ \\
\hline Quetiapine & $0.88(1)$ & $0.90(5)$ & $1.00(3)$ & $0.91(8)$ & $0.91(8)$ & $0.93(7)$ & $146(7)$ & $139(8)$ & $137(3)$ & $143(4)$ & $133(4)$ & $129(4)$ \\
\hline Risperidone & 0.85 (3) & $0.85(5)$ & $0.91(7)$ & $0.85(7)$ & 0.89 (6) & $0.91(9)$ & $116(14)$ & $107(6)$ & $125(3)$ & $123(5)$ & $117(11)$ & $114(5)$ \\
\hline
\end{tabular}


Table 4 Quantification of antipsychotics (ng/mL) using VAMS tips and plasma as sample matrix and assessment of adherence. conc. concentration

\begin{tabular}{lllll}
\hline Analyte & $\begin{array}{l}\text { Conc. in } \\
\text { VAMS }(\mathrm{ng} / \mathrm{mL})\end{array}$ & $\begin{array}{l}\text { Adherence } \\
\text { assessment } \\
\text { in VAMS }\end{array}$ & $\begin{array}{l}\text { Conc. in } \\
\text { plasma }(\mathrm{ng} / \mathrm{mL})\end{array}$ & $\begin{array}{l}\text { Adherence } \\
\text { assessment in plasma }\end{array}$ \\
\hline Amisulpride & 209 & Adherent & 216 & Adherent \\
Clozapine & 400 & Adherent & 368 & Adherent \\
Olanzapine & $93^{*}$ & Adherent & $98^{*}$ & Adherent \\
Olanzapine & 64 & Adherent & 72 & Adherent \\
Paliperidone & $<5$ & Non-adherent & 6 & Non-adherent \\
Paliperidone & 12 & Non-adherent & 12 & Non-adherent \\
Pipamperone & 75 & Non-adherent & 115 & Adherent \\
Pipamperone & $450^{*}$ & Adherent & $505^{*}$ & Adherent \\
Pipamperone & $<50$ & Non-adherent & 58 & Non-adherent \\
Pipamperone & $<50$ & Non-adherent & 15 & Non-adherent \\
Promethazine & 18 & Adherent & 10 & Adherent \\
Promethazine & 36 & Adherent & 39 & Adherent \\
Quetiapine & $<50$ & Non-adherent & 8 & Non-adherent \\
Quetiapine & $<50$ & Non-adherent & 94 & Non-adherent \\
Quetiapine & $952^{*}$ & Adherent & $1082^{*}$ & Adherent \\
Risperidone & 5 & Adherent & 10 & Adherent \\
Risperidone & 5 & Adherent & 5 & Adherent \\
\hline
\end{tabular}

*Above the therapeutic range of concept of the presented method was achieved using 10 matched human whole blood (EDTA)-loaded VAMS tips and plasma samples. Unfortunately, samples containing aripiprazole, cyamemazine, haloperidol, and prothipendyl were not available for further evaluation.

Table 4 shows the results after analyzing VAMS tips and plasma and the assessment of adherence in both matrices. For a positive assessment of adherence, the minimal concentration in blood should be above the lowest published therapeutic concentration with a tolerance limit of $\pm 15 \%$ to take measurement inaccuracy into account. Adherence assessment differed between VAMS and plasma only once. This could be explained by the measurement inaccuracy within $\pm 15 \%$ regarding plasma. In three cases, quantification revealed concentrations above the therapeutic range but it is known that high doses of antipsychotics are used for treatment [36, 37]. Results also showed that VAMS can be a promising tool for adherence monitoring and that the developed VAMS strategy is consistent with findings in plasma.

Since the method was fully validated and meets certain requirements such as a full calibration within therapeutic ranges of the antipsychotics, it might also be used for TDM. However, before using this method for TDM purpose, some more data are needed. The therapeutic ranges given in ESM Table S4 are plasma concentrations and the relationship between plasma and whole blood concentrations has to be established first. Patteet et al. calculated whole blood therapeutic ranges using blood to serum ratios and conducted a clinical study to make a comparison between antipsychotic concentrations found in venous whole blood and capillary DBS samples. They came to the result that the clinical interpretation, for the tested antipsychotics, of serum, whole blood, and DBS concentrations was identical [38]. Stern et al. also compared capillary blood, whole blood, and plasma concentrations of psychiatric drugs and came to the result that the three matrices showed a good correlation between determined concentrations [23]. However, exact concentrations including converting factors are not required for the assessment of adherence, which was the aim of the current study.

\section{Conclusions}

A VAMS- and LC-HRMS/MS-based workflow for simultaneous quantification of 13 antipsychotics was successfully developed and validated. Only $10 \mu \mathrm{L}$ of blood was required and the straightforward sample preparation was followed by a complete chromatographic separation. Proof of concept was demonstrated by quantification of 17 intakes of antipsychotics in VAMS tips and matched plasma samples of patients. VAMS as a sampling strategy for dried matrix was able to overcome the hematocrit issue in most cases but the advantage of stability in dried matrix could only be proven for half of the analytes.

Supplementary Information The online version contains supplementary material available at https://doi.org/10.1007/s00216-020-03143-0. 
Acknowledgments The authors would like to thank Armin A. Weber, Selina Hemmer, and Tanja M. Gampfer for their support.

Funding Open Access funding enabled and organized by Projekt DEAL.

\section{Compliance with ethical standards}

Conflict of interest The authors declare that they have no conflict of interest.

Open Access This article is licensed under a Creative Commons Attribution 4.0 International License, which permits use, sharing, adaptation, distribution and reproduction in any medium or format, as long as you give appropriate credit to the original author(s) and the source, provide a link to the Creative Commons licence, and indicate if changes were made. The images or other third party material in this article are included in the article's Creative Commons licence, unless indicated otherwise in a credit line to the material. If material is not included in the article's Creative Commons licence and your intended use is not permitted by statutory regulation or exceeds the permitted use, you will need to obtain permission directly from the copyright holder. To view a copy of this licence, visit http://creativecommons.org/licenses/by/4.0/.

\section{References}

1. Pfennig A, Bschor T, Falkai P, Bauer M. The diagnosis and treatment of bipolar disorder: recommendations from the current s3 guideline. Dtsch Arztebl Int. 2013;110(6):92-100. https://doi.org/ 10.3238/arztebl.2013.0092

2. Hui CLM, Lam BST, Lee EHM, Chan SKW, Chang WC, Suen $\mathrm{YN}$, et al. A systematic review of clinical guidelines on choice, dose, and duration of antipsychotics treatment in first- and multiepisode schizophrenia. Int Rev Psychiatry. 2019;31(5-6):441-59. https://doi.org/10.1080/09540261.2019.1613965.

3. Garcia S, Martinez-Cengotitabengoa M, Lopez-Zurbano S, Zorrilla I, Lopez P, Vieta E, et al. Adherence to antipsychotic medication in bipolar disorder and schizophrenic patients: a systematic review. J Clin Psychopharmacol. 2016;36(4):355-71. https://doi.org/10. 1097/JCP.0000000000000523.

4. Haddad PM, Brain C, Scott J. Nonadherence with antipsychotic medication in schizophrenia: challenges and management strategies. Patient Relat Outcome Meas. 2014;5:43-62. https://doi.org/ 10.2147/PROM.S42735.

5. Jawad I, Watson S, Haddad PM, Talbot PS, McAllister-Williams RH. Medication nonadherence in bipolar disorder: a narrative review. Ther Adv Psychopharmacol. 2018;8(12):349-63. https://doi. org/10.1177/2045125318804364.

6. Stephenson JJ, Tunceli O, Gu T, Eisenberg D, Panish J, Crivera C, et al. Adherence to oral second-generation antipsychotic medications in patients with schizophrenia and bipolar disorder: physicians' perceptions of adherence vs. pharmacy claims. Int J Clin Pract. 2012;66(6):565-73. https://doi.org/10.1111/j.1742-1241. 2012.02918.x.

7. Bohlken J, Konrad M, Kostev K. Adherence to neuroleptic treatment in psychiatric practices: a retrospective study of 55 practices with more than 5000 bipolar and schizophrenic patients in Germany. Psychiatry Res. 2020;284:112758. https://doi.org/10. 1016/j.psychres.2020.112758.

8. Higashi K, Medic G, Littlewood KJ, Diez T, Granstrom O, De Hert M. Medication adherence in schizophrenia: factors influencing adherence and consequences of nonadherence, a systematic literature review. Ther Adv Psychopharmacol. 2013;3(4):200-18. https://doi. org/10.1177/2045125312474019.

9. Keefe RS, Bilder RM, Davis SM, Harvey PD, Palmer BW, Gold $\mathrm{JM}$, et al. Neurocognitive effects of antipsychotic medications in patients with chronic schizophrenia in the CATIE trial. Arch Gen Psychiatry. 2007;64(6):633-47. https://doi.org/10.1001/archpsyc. 64.6.633.

10. Hiemke C, Bergemann N, Clement HW, Conca A, Deckert J, Domschke K, et al. Consensus guidelines for therapeutic drug monitoring in neuropsychopharmacology: update 2017. Pharmacopsychiatry. 2017. https://doi.org/10.1055/s-0043116492.

11. Vieta E, Locklear J, Gunther O, Ekman M, Miltenburger C, Chatterton ML, et al. Treatment options for bipolar depression: a systematic review of randomized, controlled trials. J Clin Psychopharmacol. 2010;30(5):579-90. https://doi.org/10.1097/ JCP.0b013e3181f15849.

12. Guthrie R, Susi A. A simple phenylalanine method for detecting phenylketonuria in large populations of newborn infants. Pediatrics. 1963;32:338-43.

13. Edelbroek PM, van der Heijden J, Stolk LM. Dried blood spot methods in therapeutic drug monitoring: methods, assays, and pitfalls. Ther Drug Monit. 2009;31(3):327-36. https://doi.org/10. 1097/FTD.0b013e31819e91ce.

14. Patteet L, Maudens KE, Stove CP, Lambert WE, Morrens M, Sabbe $\mathrm{B}$, et al. The use of dried blood spots for quantification of 15 antipsychotics and 7 metabolites with ultra-high performance liquid chromatography - tandem mass spectrometry. Drug Test Anal. 2015;7(6):502-11. https://doi.org/10.1002/dta.1698.

15. De Kesel PM, Sadones N, Capiau S, Lambert WE, Stove CP. Hemato-critical issues in quantitative analysis of dried blood spots: challenges and solutions. Bioanalysis. 2013;5(16):2023-41. https:// doi.org/10.4155/bio.13.156.

16. Denniff P, Spooner N. Volumetric absorptive microsampling: a dried sample collection technique for quantitative bioanalysis. Anal Chem. 2014;86(16):8489-95. https://doi.org/10.1021/ ac5022562.

17. Spooner N, Denniff P, Michielsen L, De Vries R, Ji QC, Arnold $\mathrm{ME}$, et al. A device for dried blood microsampling in quantitative bioanalysis: overcoming the issues associated blood hematocrit. Bioanalysis. 2015;7(6):653-9. https://doi.org/10.4155/bio.14.310.

18. Verougstraete N, Lapauw B, Van Aken S, Delanghe J, Stove C, Stove V. Volumetric absorptive microsampling at home as an alternative tool for the monitoring of $\mathrm{HbAl} \mathrm{c}$ in diabetes patients. Clin Chem Lab Med. 2017;55(3):462-9. https://doi.org/10.1515/cclm2016-0411.

19. Volani C, Caprioli G, Calderisi G, Sigurdsson BB, Rainer J, Gentilini I, et al. Pre-analytic evaluation of volumetric absorptive microsampling and integration in a mass spectrometry-based metabolomics workflow. Anal Bioanal Chem. 2017;409(26):6263-76. https://doi.org/10.1007/s00216-017-0571-8.

20. Mercolini L, Protti M, Catapano MC, Rudge J, Sberna AE. LC-MS/ MS and volumetric absorptive microsampling for quantitative bioanalysis of cathinone analogues in dried urine, plasma and oral fluid samples. J Pharm Biomed Anal. 2016;123:186-94. https://doi. org/10.1016/j.jpba.2016.02.015.

21. Protti M, Catapano MC, Samolsky Dekel BG, Rudge J, Gerra G, Somaini L, et al. Determination of oxycodone and its major metabolites in haematic and urinary matrices: comparison of traditional and miniaturised sampling approaches. J Pharm Biomed Anal. 2018;152:204-14. https://doi.org/10.1016/j.jpba.2018.01.043.

22. Kok MGM, Fillet M. Volumetric absorptive microsampling: current advances and applications. J Pharm Biomed Anal. 2018;147: 288-96. https://doi.org/10.1016/j.jpba.2017.07.029.

23. Stern M, Giebels M, Fey T, Lubking M, Alferink J, Hempel G. Validation and clinical application of a volumetric absorptive 
microsampling method for 14 psychiatric drugs. Bioanalysis. 2020;12(16):1129-47. https://doi.org/10.4155/bio-2020-0136.

24. Ritscher S, Hoyer M, Georges C, Wunder C, Wallemacq P, Persu A, et al. Benefit of serum drug monitoring complementing urine analysis to assess adherence to antihypertensive drugs in first-line therapy. PLoS One. 2020;15(8):e0237383. https://doi.org/10.1371/ journal.pone. 0237383 .

25. Guideline on bioanalytical method validation, EMEA/CHMP/ EWP/192217/2009 Rev.1 Corr.2 (2011).

26. Capiau S, Veenhof H, Koster RA, Bergqvist Y, Boettcher M, Halmingh $\mathrm{O}$, et al. Official International Association for Therapeutic Drug Monitoring and Clinical Toxicology Guideline: development and validation of dried blood spot-based methods for therapeutic drug monitoring. Ther Drug Monit. 2019;41(4):40930. https://doi.org/10.1097/FTD.0000000000000643.

27. Koster RA, Alffenaar JW, Botma R, Greijdanus B, Touw DJ, Uges $\mathrm{DR}$, et al. What is the right blood hematocrit preparation procedure for standards and quality control samples for dried blood spot analysis? Bioanalysis. 2015;7(3):345-51. https://doi.org/10.4155/bio. 14.298.

28. DIN. Haematology - Procedure for determining the volume fraction of erythrocytes (packed cell volume) in blood - Part 1. Reference method based on centrifugation. DIN 58933-1:1995-01 1995. https://doi.org/10.31030/2715960.

29. Koster RA, Niemeijer P, Veenhof H, Hateren KV, Alffenaar JC, Touw DJ. A volumetric absorptive microsampling LC-MS/MS method for five immunosuppressants and their hematocrit effects. Bioanalysis. 2019;11(6):495-508. https://doi.org/10.4155/bio2018-0312.

30. D'Urso A, Rudge J, Patsalos PN, de Grazia U. Volumetric absorptive microsampling: a new sampling tool for therapeutic drug monitoring of antiepileptic drugs. Ther Drug Monit. 2019;41(5):68192. https://doi.org/10.1097/FTD.0000000000000652.

31. Montenarh D, Hopf M, Maurer HH, Schmidt P, Ewald AH. Development and validation of a multi-analyte LC-MS/MS approach for quantification of neuroleptics in whole blood, plasma, and serum. Drug Test Anal. 2016;8(10):1080-9. https://doi.org/10. 1002/dta.1923.

32. Maurer HH, Meyer MR, Helfer AG, Weber AA. Maurer/Meyer/ Helfer/Weber MMHW LC-HR-MS/MS library of drugs, poisons, and their metabolites. Weinheim: Wiley-VCH; 2018.

33. Saar E, Gerostamoulos D, Drummer OH, Beyer J. Assessment of the stability of 30 antipsychotic drugs in stored blood specimens. Forensic Sci Int. 2012;215(1-3):152-8. https://doi.org/10.1016/j. forsciint.2011.02.022.

34. Tron C, Kloosterboer SM, van der Nagel BCH, Wijma RA, Dierckx B, Dieleman GC, et al. Dried blood spots combined with ultra-high-performance liquid chromatography-mass spectrometry for the quantification of the antipsychotics risperidone, aripiprazole, pipamperone, and their major metabolites. Ther Drug Monit. 2017;39(4):429-40. https://doi.org/10.1097/FTD. 0000000000000411.

35. Geers LM, Cohen D, Wehkamp LM, van Hateren K, Koster RA, Fedorenko OY, et al. Dried blood spot analysis for therapeutic drug monitoring of clozapine. J Clin Psychiatry. 2017;78(9):e1211-e8. https://doi.org/10.4088/JCP.16m11164.

36. Sernyak MJ, Rosenheck R. Clinicians' reasons for deviations from recommended dosing practices for antipsychotic medications. Admin Pol Ment Health. 2007;34(6):540-7. https://doi.org/10. 1007/s10488-007-0142-y.

37. Hanssens L, De Hert M, Wampers M, Reginster JY, Peuskens J. Pharmacological treatment of ambulatory schizophrenic patients in Belgium. Clin Pract Epidemiol Ment Health. 2006;2:11. https://doi. org/10.1186/1745-0179-2-11.

38. Patteet L, Maudens KE, Stove CP, Lambert WE, Morrens M, Sabbe B, et al. Are capillary DBS applicable for therapeutic drug monitoring of common antipsychotics? A proof of concept. Bioanalysis. 2015;7(16):2119-30. https://doi.org/10.4155/bio.15.100.

Publisher's note Springer Nature remains neutral with regard to jurisdictional claims in published maps and institutional affiliations. 